\title{
A TRANSLATION PRINCIPLE FOR KAC-MOODY ALGEBRAS
}

\author{
WAYNE NEIDHARDT
}

\begin{abstract}
Let $\mathfrak{g}$ be a Kac-Moody algebra defined by a symmetrizable generalized Cartan matrix. We show that the multiplicity of the irreducible module $L\left(w_{1} \cdot \lambda\right)$ in the Verma module $M\left(w_{2} \cdot \lambda\right)$ depends only on the elements $w_{1}$ and $w_{2}$ of the Weyl group, and not on the dominant integral weight $\lambda$, generalizing the translation principle of Jantzen for finite-dimensional algebras.
\end{abstract}

1. Introduction. In [3], Jantzen proves a translation principle for a finitedimensional split semisimple Lie algebra $\mathfrak{g}$ over a field of characteristic zero. This implies [3, Corollary 2.15] that the multiplicity $\left(M\left(w_{1} \cdot \lambda\right): L\left(w_{2} \cdot \lambda\right)\right)$ of the irreducible module $L\left(w_{2} \cdot \lambda\right)$ of highest weight $w_{2} \cdot \lambda$ in the Verma module $M\left(w_{1} \cdot \lambda\right)$ of highest weight $w_{1} \cdot \lambda$ is independent of the choice of dominant integral weight $\lambda$, i.e. the multiplicity depends only on the elements $w_{1}$ and $w_{2}$ of the Weyl group. We show that this result holds for the case where $\mathfrak{g}$ is defined by a symmetrizable generalized Cartan matrix. This is the content of Corollary 5.5.

The idea of the proof follows the same general line of argument as that of Jantzen, but there are several difficulties in the infinite-dimensional case. One is that in the definition of the translation functors, we must tensor with a module whose set of weights is stable under the Weyl group. Since not all integral weights are conjugate to dominant integral weights, we must restrict ourselves to certain translations. For this reason, we only translate within the chamber, and do not consider the facettes in its closure. This simplifies matters somewhat by restricting the problem, but does not help with the solution. Even within the dominant chamber, not all translations are possible, so we must first observe that it is enough to obtain the result for translation from 0 to an arbitrary dominant integral weight. Next, to show what translation does to a Verma module, we apply the Casimir operator (which plays the role of central characters) and select one of the generalized eigenspaces. It is not true in general that two highest weights with the same generalized eigenvalue are in the same Weyl orbit, so care must be taken in the proof of Proposition 3.3 to show that in this case we need only consider one Weyl orbit. Finally, Jantzen's proof that the different generalized eigenspaces are orthogonal with respect to a contravariant form does not work in this case, and we use instead a g-module structure on the $U(\mathfrak{h})$-finite part of the dual of a weight module defined by an involutive antiautomorphism of $\mathfrak{g}$ to obtain this orthogonality in Proposition 4.4.

In $\S 2$, we give the basic definitions and notation associated with Kac-Moody algebras. We introduce the Casimir operators in $\S 3$ and use them to define the translation functors. Contravariant forms are discussed in $\S 4$, where we prove a

Received by the editors January 28, 1986.

1980 Mathematics Subject Classification (1985 Revision). Primary 17B10, 17B65.

(C) 1987 American Mathematical Society $0002-9939 / 87 \$ 1.00+\$ .25$ per page 
property relating Casimir operators and contravariant forms. The main result is proved in $\S 5$.

2. Preliminaries. Let $K$ be a field of characteristic zero, and let $\mathfrak{g}$ be the KacMoody albegra over $K$ defined by a symmetrizable $\ell \times \ell$ generalized Cartan matrix $A=\left(A_{i j}\right)$, so that $\mathfrak{g}$ is generated by elements $e_{1}, \ldots, e_{\ell}$ and $f_{1}, \ldots, f_{\ell}$, together with its Cartan subalgebra, $\mathfrak{h}$, which is of dimension $2 \ell-\operatorname{rank} A$ (cf. [4 and 7 ]). Let $\left\{\alpha_{1}, \ldots, \alpha_{\ell}\right\}$ be the set of simple roots, a linearly independent subset of $\mathfrak{h}^{*}$, the dual of $\mathfrak{h}$, such that $\left[h, e_{i}\right]=\alpha_{i}(h) e_{i}$ and $\left[h, f_{i}\right]=-\alpha_{i}(h) f_{i}$ for all $h \in \mathfrak{h}$ and all $i=1, \ldots, \ell$. Let $\left\{h_{1}, \ldots, h_{\ell}\right\}$ be the set of simple coroots, a linearly independent subset of $\mathfrak{h}$ such that $\left[e_{i}, f_{j}\right]=\delta_{i j}{ }^{\prime} h_{i}$ and $\alpha_{i}\left(h_{j}\right)=A_{j i}$ for all $i$ and $j$. In particular, $\alpha_{i}\left(h_{i}\right)=2$ for $i=1, \ldots, \ell$.

The symmetrizability of $A$ gaurantees the existence of a nondegenerate invariant symmetric bilinear form $\langle-,-\rangle$ on $\mathfrak{g}$, whose restriction to $\mathfrak{h}$ is again nondegenerate, hence induces a nondegenerate bilinear form on $\mathfrak{h}^{*}$, which we also denote by $\langle-,-\rangle$.

For each $i=1, \ldots, \ell$, define the linear involution $r_{i}$ on $\mathfrak{h}^{*}$ by $r_{i}(\lambda)=\lambda-\lambda\left(h_{i}\right) \alpha_{i}$ for any $\lambda \in \mathfrak{h}^{*}$, and define the Weyl group $W$ of $\mathfrak{g}$ to be the subgroup of GL( $\left(\mathfrak{h}^{*}\right)$ generated by $\left\{r_{1}, \ldots, r_{\ell}\right\}$. Fix $\rho \in \mathfrak{h}^{*}$ such that $\rho\left(h_{i}\right)=1$ for all $i=1, \ldots, \ell$. Then we also have a "dot" action of $W$ on $\mathfrak{h}^{*}$ given by $w \cdot \lambda=w(\lambda+\rho)-\rho$ for all $w \in W$, $\lambda \in \mathfrak{h}^{*}$.

If $M$ is an $\mathfrak{h}$-module and $\lambda \in \mathfrak{h}^{*}$, let $M_{\lambda}=\{m \in M \mid h \cdot m=\lambda(h) m$ for all $h \in \mathfrak{h}\}$, called the $\lambda$-weight space of $M$. If $M_{\lambda} \neq 0$, we call $\lambda$ a weight of $M$, and we let $\Pi(M)$ denote the set of weights of $M$. In case $M=\bigoplus_{\lambda \in \mathfrak{h}^{*}} M_{\lambda}$ with all $M_{\lambda}$ finite-dimensional, we call $M$ a weight module. In particular, with the adjoint action, we have $\mathfrak{g}=\bigoplus_{\alpha \in \mathfrak{h}^{*}} \mathfrak{g}_{\alpha}$. The roots of $\mathfrak{g}$ are those $\alpha \in \mathfrak{h}^{*} \backslash\{0\}$ such that $\mathfrak{g}_{\alpha} \neq 0$. We let $\Delta$ denote the set of roots. Then $\Delta=\Delta^{+} \cup \Delta^{-}$, where $\Delta^{+}$is the set of positive roots relative to $\left\{\alpha_{1}, \ldots, \alpha_{\ell}\right\}$ and $\Delta^{-}$is the set of negative roots.

Let $\mathfrak{n}^{+}$be the subalgebra of $\mathfrak{g}$ generated by $\left\{e_{1}, \ldots, e_{\ell}\right\}$, and let $\mathfrak{b}=\mathfrak{h} \oplus \mathfrak{n}^{+}$. If $M$ is a $\mathfrak{g}$-module, $\lambda \in \mathfrak{h}^{*}$, and there is a nonzero vector $v \in M_{\lambda}$ such that $\mathfrak{n}^{+} \cdot v=0$ and $M=U(\mathfrak{g}) v$, then we call $M$ a highest weight module of weight $\lambda$.

For any $\lambda \in \mathfrak{h}^{*}$, let $K(\lambda)$ be the one-dimensional $\mathfrak{b}$-module which is $\mathfrak{n}^{+}$-trivial and whose unique weight is $\lambda$. The induced module $M(\lambda)=U(\mathfrak{g}) \otimes_{U(\mathfrak{b})} K(\lambda)$ is called the Verma module of weight $\lambda$. It is well known that $M(\lambda)$ is the universal highest weight module of weight $\lambda$, and that $M(\lambda)$ has a unique irreducible quotient, which we denote by $L(\lambda)$, and which is also of highest weight $\lambda$.

Let $P^{+}=\left\{\lambda \in \mathfrak{h}^{*} \mid \lambda\left(h_{i}\right)\right.$ is a nonnegative integer for all $\left.i=1, \ldots, \ell\right\}$. It is easy to see that when $\lambda \in P^{+}, \Pi(L(\lambda))$ is invariant under $W$; in fact $\operatorname{dim} L(\lambda)_{\mu}=$ $\operatorname{dim} L(\lambda)_{w \mu}$ for all $\mu \in \mathfrak{h}^{*}$ and $w \in W$ when $\lambda \in P^{+}$.

We partially order $\mathfrak{h}^{*}$ by $\mu \leq \lambda$ if $\lambda-\mu$ is a nonnegative integral linear combination of the simple roots $\alpha_{1}, \ldots, \alpha_{\ell}$. If $\lambda \in \mathfrak{h}^{*}$, let $C(\lambda)$ denote the full subcategory of the category of $\mathfrak{g}$-modules consisting of those modules $M$ which are weight modules such that $\mu \in \Pi(M)$ implies $\mu \leq \lambda$ (cf. [8]).

We will need certain types of filtrations of modules in $C(\lambda)$ by submodules.

DEFINITION 2.1. Let $M$ be an object in $C(\lambda)$. By a highest weight series (HWS) for $M$, we mean a filtration $0=M_{0} \subset M_{1} \subset \cdots$ of $M$ by submodules such that $M=\bigcup_{i \geq 0} M_{i}$ and each factor $F_{i}=M_{i} / M_{i-1}$ is a highest weight module.

DEFINITION 2.2. Let $M$ be an object in $C(\lambda)$. By a Verma Series (VS) for $M$, we mean a filtration $0=M_{0} \subset M_{1} \subset \cdots$ of $M$ by submodules such that 
$M=\bigcup_{i \geq 0} M_{i}$ and each factor $F_{i}=M_{i} / M_{i-1}$ is isomorphic to some $M\left(\lambda_{i}\right)$. In case $M$ has a VS, we write $(M: M(\mu))$ for the number of occurrences of $M(\mu)$ as a factor. This is independent of the choice of VS for $M$.

REMARK. Any VS is a HWS, but although any object in $C(\lambda)$ has a HWS, it does not necessarily have a VS.

Observe that if $L$ is an object in $C(\lambda-\mu)$, then $M(\mu) \otimes_{K} L$ is an object in $C(\lambda)$. The following result is fairly standard.

PROPOSITION 2.3 [2, PROPOSITION 1.10]. Let $\mu \in \mathfrak{h}^{*}$ and $L$ be an object in $C(\lambda-\mu)$. Then $M(\mu) \otimes_{K} L$ has a VS, and $\left(M(\mu) \otimes_{K} L: M(\nu)\right)=\operatorname{dim} L_{\nu-\mu}$ for any $\nu \in \mathfrak{h}^{*}$.

DEFINITION $2.4[\mathbf{1}]$. Let $M$ be an object of $C(\lambda)$, and let $\mu \in \mathfrak{h}^{*}$. By a local composition series (LCS) for $M$ at $\mu$, we mean a finite filtration $0=M_{0} \subset M_{1} \subset$ $\cdots \subset M_{n}=M$ of $M$ by submodules such that each factor $F_{i}=M_{i} / M_{i-1}$ either has no weights $\geq \mu$ or is isomorphic to some $L\left(\lambda_{i}\right)$. We write $(M: L(\mu))$ for the number of occurrences of $L(\mu)$ as a factor in any LCS for $M$ at $\mu$ (or at any $\nu \leq \mu$ ), which is independent of the choice of LCS.

REMARK. For any object $M$ in $C(\lambda)$ and any $\mu \in \mathfrak{h}^{*}$, an LCS for $M$ at $\mu$ always exists. Also, it is clear that $(M: L(\mu)) \neq 0$ if and only if $L(\mu)$ is a subquotient of M.

3. Casimir operators and translation functors. Fix $\lambda \in \mathfrak{h}^{*}$. For each $\alpha \in \Delta^{+}$, let $\left\{e_{\alpha}^{(1)}, \ldots, e_{\alpha}^{(m(\alpha))}\right\}$ be a basis for $\mathfrak{g}_{\alpha}$, where $m(\alpha)=\operatorname{dim} \mathfrak{g}_{\alpha}$, and let $\left\{f_{\alpha}^{(1)}, \ldots, f_{\alpha}^{(m(\alpha))}\right\}$ be the dual basis of $\mathfrak{g}_{-\alpha}$ with respect to the form $\langle-,-\rangle$. For any object $M$ in $C(\lambda)$, it is clear that

$$
\Gamma_{1, M}=\sum_{\alpha \in \Delta^{+}} \sum_{i=1}^{m(\alpha)} f_{\alpha}^{(i)} e_{\alpha}^{(i)}
$$

acts as a well-defined linear transformation on $M$. Define $\Gamma_{2, M}$ so that $\Gamma_{2, M}$ acts on $M_{\nu}$ by the scalar $\langle\nu+\rho, \nu+\rho\rangle$ for each $\nu \in \Pi(M)$. Then $\Gamma_{M}=\Gamma_{1, M}+\Gamma_{2, M}$ is called the Casimir operator on $M$. These operators were introduced in [5]. It is well known that in case $M$ is a highest weight module of weight $w \cdot \nu$ for some $w \in W$ and $\nu \in \mathfrak{h}^{*}, \Gamma_{M}$ acts as the scalar $\langle\nu+\rho, \nu+\rho\rangle$ on $M$.

Proposition 3.1 [2, Propositions 4.5 AND 4.7]. For any object $M$ in $C(\lambda)$ and any $k \in K$, let $M^{(k)}$ be the generalized eigenspace in $M$ for the eigenvalue $k$ of $\Gamma_{M}$. Then $M=\bigoplus_{k \in K} M^{(k)}$. Furthermore, if $0=M_{0} \subset M_{1} \subset \cdots$ is a HWS for $M$, where each factor $F_{i}=M_{i} / M_{i-1}$ is of highest weight $\lambda_{i}$, then $M^{(k)}$ has a $H W S$ whose factors are precisely those $F_{i}$ such that $\left\langle\lambda_{i}+\rho, \lambda_{i}+\rho\right\rangle=k$, in order. For any $\mathfrak{g}$-module homomorphism $\phi: M \rightarrow N$ in $C(\lambda), \phi\left(M^{(k)}\right) \subseteq N^{(k)}$, and the assignment $M \mapsto M^{(k)}$ together with restriction of maps is an exact functor on $C(\lambda)$.

Definition $3.2[3, \S 2.10]$. For any $\lambda, \mu \in \mathfrak{h}^{*}$, define the exact functor $T_{\mu}^{\lambda}$ from $C(\mu)$ to $C(\lambda)$ by

$$
T_{\mu}^{\lambda} M=\left(M \otimes_{K} L(\lambda-\mu)\right)^{(\langle\lambda+\rho, \lambda+\rho\rangle)},
$$

called the translation from $\mu$ to $\lambda$. 
REMARK. In [3], the translation $T_{\mu}^{\lambda}$ is only defined for those $M$ such that $M=M^{(\langle\mu+\rho, \mu+\rho\rangle)}$, and when $\lambda-\mu$ is an integral weight. Although we will only apply the functors in this case, it is not necessary for our purposes to make such restrictions.

Proposition 3.3. Let $\lambda \in P^{+}, w \in W$. Then $T_{0}^{\lambda} M(w \cdot 0) \cong M(w \cdot \lambda)$.

ProOF. By Proposition 2.3, $M(w \cdot 0) \otimes_{K} L(\lambda)$ has a VS with

$$
\left(M(w \cdot 0) \otimes_{K} L(\lambda): M(\mu)\right)=\operatorname{dim} L(\lambda)_{\mu-w \cdot 0}
$$

for any $\mu \in \mathfrak{h}^{*}$. Thus, if $M(\mu)$ occurs as a factor, then $\mu=w \cdot 0+\chi$ for some $\chi \in \Pi(L(\lambda))$. Now, $W \cdot 0+\Pi(L(\lambda))$ is stable under the dot action of $W$, and all its elements are $\leq \lambda$, so that by $\left[2\right.$, Proposition 2.9], $\mu=w^{\prime} \cdot \nu$ for some $w^{\prime} \in W$ and $\nu \in(W \cdot 0+\Pi(L(\lambda)))$ with $\nu+\rho \in P^{+}$. (Just choose $\nu \in W \cdot \mu$ with $\lambda-\nu$ minimal.) Now $T_{0}^{\lambda} M(w \cdot 0)$ has a VS whose factors are those $M(\mu)$ with $\mu=w^{\prime} \cdot \nu$ as described above with the additional condition that $\langle\mu+\rho, \mu+\rho\rangle=\langle\lambda+\rho, \lambda+\rho\rangle$. But then $\langle\nu+\rho, \nu+\rho\rangle=\langle\lambda+\rho, \lambda+\rho\rangle$ and $\nu \leq \lambda$ with $\lambda \in P^{+}$and $\nu+\rho \in P^{+}$. By [2, Proposition 2.12], $\nu=\lambda$. Thus $T_{0}^{\lambda} M(w \cdot 0)$ has a VS whose factors are all of the form $M\left(w^{\prime} \cdot \lambda\right)$ with $w^{\prime} \in W$. But now, we have

$$
\begin{aligned}
\left(T_{0}^{\lambda} M(w \cdot 0): M\left(w^{\prime} \cdot \lambda\right)\right) & =\operatorname{dim} L(\lambda)_{w^{\prime} \cdot \lambda-w \cdot 0}=\operatorname{dim} L(\lambda)_{\left(w^{\prime}\right)^{-1}\left(w^{\prime} \cdot \lambda-w \cdot 0\right)} \\
& =\operatorname{dim} L(\lambda)_{\lambda-\left(w^{\prime}\right)^{-1} w \cdot 0}=\delta_{w^{\prime}, w},
\end{aligned}
$$

since $\left(w^{\prime}\right)^{-1} w \cdot 0<0$ whenever $w^{\prime} \neq w$. Thus the VS has only one factor, isomorphic to $M(w \cdot \lambda)$.

4. Contravariant forms. Let $\sigma: \mathfrak{g} \rightarrow \mathfrak{g}$ be the involutive antiautomorphism defined by $\sigma\left(e_{i}\right)=f_{i}$ for $i=1, \ldots, \ell$ and $\sigma(h)=h$ for all $h \in \mathfrak{h}$. This induces an antiautomorphism of $U(\mathfrak{g})$, which we denote also by $\sigma$.

DEFINITION 4.1. A bilinear form $(-,-)$ on a $\mathfrak{g}$-module $M$ is said to be contravariant if $\left(u m_{1}, m_{2}\right)=\left(m_{1}, \sigma(u) m_{2}\right)$ for all $u \in U(\mathfrak{g})$ and all $m_{1}, m_{2} \in M$.

PROPOSITION 4.2 [3, SATZ 1.6(b)]. Let $M$ be a highest weight module. Then $M$ has a nonzero contravariant form, uniquely determined up to scalar multiples, and this form is nondegenerate if and only if $M$ is irreducible.

For the next proposition, we will need to define a $\mathfrak{g}$-module structure on the dual of a weight module which differs from the usual structure. The basic properties of this $\mathfrak{g}$-module structure are noted in [1].

DEFINITION 4.3. Let $M$ be a weight module. Define $M^{\sigma}$ to be the $\mathfrak{g}$-module whose underlying vector space is the $U(\mathfrak{h})$-finite part of $M^{*}$, with $\mathfrak{g}$-module action defined by $(x \cdot f)(m)=f(\sigma(x) \cdot m)$ for all $x \in \mathfrak{g}, f \in M^{\sigma}$, and $m \in M$.

$M \mapsto M^{\sigma}$, together with transposition of $\mathfrak{g}$-module homomorphisms, defines an exact, contravariant functor on $C(\lambda)$ for any $\lambda \in \mathfrak{h}^{*}$. Using this fact, we see that for any LCS for $M$, there is an LCS for $M^{\sigma}$ with the order of the irreducible factors reversed. In particular, $(M: L(\mu))=\left(M^{\sigma}, L(\mu)\right)$ for any $\mu \in \mathfrak{h}^{*}$.

Proposition 4.4. Let $\lambda \in \mathfrak{h}^{*}$, and let $M$ be an object in $C(\lambda)$. Suppose $M$ has a contravariant form $(-,-)$. Then the various $M^{(k)}, k \in K$, are pairwise orthogonal.

PrOOF. Suppose $k \neq k^{\prime}$, and consider the restriction of the form to $M^{(k)} \times$ $M^{\left(k^{\prime}\right)}$. For any $m \in M^{(k)}$, the map $(m,-): M^{\left(k^{\prime}\right)} \rightarrow K$ is in the $U(\mathfrak{h})$-finite part 
of $M^{\left(k^{\prime}\right)^{*}}$, and in fact the assignment $m \mapsto(m,-)$ is a $\mathfrak{g}$-module homomorphism $\phi: M^{(k)} \rightarrow M^{\left(k^{\prime}\right) \sigma}$, by the contravariance of the form. Now, any irreducible subquotient $L(\mu)$ of $M^{\left(k^{\prime}\right) \sigma}$ is also a subquotient of $M^{\left(k^{\prime}\right)}$ by the above remarks, so that $\langle\mu+\rho, \mu+\rho\rangle=k^{\prime}$. This implies that $M^{\left(k^{\prime}\right) \sigma}=\left(M^{\left(k^{\prime}\right) \sigma}\right)^{\left(k^{\prime}\right)}$. On the other hand, the functoriality in Proposition 3.1 implies that $\phi\left(M^{(k)}\right) \subseteq\left(M^{\left(k^{\prime}\right) \sigma}\right)^{(k)}$. Since $\left(M^{\left(k^{\prime}\right) \sigma}\right)^{(k)} \cap\left(M^{\left(k^{\prime}\right) \sigma}\right)^{\left(k^{\prime}\right)}=0$ by the directness of the decomposition in Proposition 3.1 , we must have $\left(M^{\left(k^{\prime}\right) \sigma}\right)^{(k)}=0$, and hence $\phi=0$, which proves the proposition.

5. The translation principle. The following lemma is the key to the main theorem. The proof is based on that of [3, Satz 2.4], but we need Proposition 4.4 for the orthogonality, along with a consequence of Proposition 3.3.

LEMMA 5.1. Let $\lambda \in P^{+}, w \in W$. Then $T_{0}^{\lambda} L(w \cdot 0) \cong L(w \cdot \lambda)$ or $T_{0}^{\lambda} L(w \cdot 0)=0$.

ProOF. Since $L(w \cdot 0)$ and $L(\lambda)$ have nondegenerate contravariant forms, so does $L(w \cdot 0) \otimes_{K} L(\lambda)$. (Simply take the tensor product of the two forms.) Since the various $\left(L(w \cdot 0) \otimes_{K} L(\lambda)\right)^{(k)}, k \in K$, are pairwise orthogonal by Proposition 4.4 , we have that the restriction of this form to

$$
T_{0}^{\lambda} L(w \cdot 0)=\left(L(w \cdot 0) \otimes_{K} L(\lambda)\right)^{(\langle\lambda+\rho, \lambda+\rho\rangle)}
$$

is nondegenerate. But $L(w \cdot 0)$ is a quotient of $M(w \cdot 0)$ and $T_{0}^{\lambda}$ is exact, so $T_{0}^{\lambda} L(w \cdot 0)$ is a quotient of $T_{0}^{\lambda} M(w \cdot 0) \cong M(w \cdot \lambda)$, by Proposition 3.3. Thus $T_{0}^{\lambda} L(w \cdot 0)$ is either zero or a highest weight module of weight $w \cdot \lambda$. Since it has a nondegenerate contravariant form, the result follows from Proposition 4.2.

LEMMA 5.2. Let $\lambda \in P^{+}, \mu \in \mathfrak{h}^{*}$, and $w \in W$. If $0=M_{0} \subset M_{1} \subset \cdots \subset$ $M_{n}=M(w \cdot 0)$ is an $L C S$ for $M(w \cdot 0)$ at $\mu-\lambda$, then $0=T_{0}^{\lambda} M_{0} \subset T_{0}^{\lambda} M_{1} \subset \cdots \subset$ $T_{0}^{\lambda} M_{n} \cong M(w \cdot \lambda)$ is an LCS for $M(w \cdot \lambda)$ at $\mu$.

PROOF. Since $T_{0}^{\lambda}$ is an exact functor, $T_{0}^{\lambda} M_{i} / T_{0}^{\lambda} M_{i-1} \cong T_{0}^{\lambda}\left(M_{i} / M_{i-1}\right)$ for each $i=1, \ldots, n$. If $M_{i} / M_{i-1}$ has no weights $\geq \mu-\lambda$, then $T_{0}^{\lambda}\left(M_{i} / M_{i-1}\right) \subseteq$ $\left(M_{i} / M_{i-1}\right) \otimes_{K} L(\lambda)$ has no weights $\geq \mu$. On the other hand, if $M_{i} / M_{i-1}$ is irreducible, it is of the form $L\left(w^{\prime} \cdot 0\right)$ for some $w^{\prime} \in W$ by [6, Theorem 4.2 and 8 , Lemma 8.2], and hence $T_{0}^{\lambda}\left(M_{i} / M_{i-1}\right)$ is irreducible or zero by Lemma 5.1.

THEOREM 5.3. Let $\lambda \in P^{+}$. Then for any $w_{1}, w_{2} \in W$,

$$
\left(M\left(w_{1} \cdot \lambda\right): L\left(w_{2} \cdot \lambda\right)\right)=\left(M\left(w_{1} \cdot 0\right): L\left(w_{2} \cdot 0\right)\right)
$$

ProOF. Let $0=M_{0} \subset M_{1} \subset \cdots \subset M_{n}=M\left(w_{1} \cdot 0\right)$ be an LCS for $M\left(w_{1} \cdot 0\right)$ at $w_{2} \cdot 0+w_{2} \lambda-\lambda=w_{2} \cdot \lambda-\lambda$. Note that $w_{2} \lambda-\lambda \leq 0$, so that this is also an LCS at $w_{2} \cdot 0$, and the number of occurrences of $L\left(w_{2} \cdot 0\right)$ as a factor is $\left(M\left(w_{1} \cdot 0\right): L\left(w_{2} \cdot 0\right)\right)$. Applying $T_{0}^{\lambda}$, we obtain an LCS for $M\left(w_{1} \cdot \lambda\right)$ at $w_{2} \cdot \lambda$ by Lemma 5.2 , and by Lemma 5.1 the number of occurrences of $L\left(w_{2} \cdot \lambda\right)$ as a factor is either 0 in case $T_{0}^{\lambda} L\left(w_{2} \cdot 0\right)$ is zero, or $\left(M\left(w_{1} \cdot 0\right): L\left(w_{2} \cdot 0\right)\right)$ in case $T_{0}^{\lambda} L\left(w_{2} \cdot 0\right) \cong L\left(w_{2} \cdot \lambda\right)$. But, by [8, Theorem 8.15(i)], $\left(M\left(w_{1} \cdot 0\right): L\left(w_{2} \cdot 0\right)\right) \neq 0$ if and only if $w_{1} \leq w_{2}$ in the Bruhat order on $W$, and likewise $\left(M\left(w_{1} \cdot \lambda\right): L\left(w_{2} \cdot \lambda\right)\right) \neq 0$ if and only if $w_{1} \leq w_{2}$. Thus $\left(M\left(w_{1} \cdot 0\right): L\left(w_{2} \cdot 0\right)\right) \neq 0$ if and only if $\left(M\left(w_{1} \cdot \lambda\right): L\left(w_{2} \cdot \lambda\right)\right) \neq 0$, and the result follows. 
COROLlaRY 5.4. Let $\lambda \in P^{+}$. Then $T_{0}^{\lambda} L(w \cdot 0) \cong L(w \cdot \lambda)$ for all $w \in W$.

ProOF. Take $w=w_{1}=w_{2}$ in the proof of the theorem, so that

$$
\left(M\left(w_{1} \cdot 0\right): L\left(w_{2} \cdot 0\right)\right)=(M(w \cdot 0): L(w \cdot 0))=1 \neq 0 .
$$

By the proof of the theorem,

$$
(M(w \cdot \lambda): L(w \cdot \lambda))= \begin{cases}0 & \text { if } T_{0}^{\lambda} L(w \cdot 0)=0, \\ (M(w \cdot 0): L(w \cdot 0)) & \text { if } T_{0}^{\lambda} L(w \cdot 0) \cong L(w \cdot \lambda) .\end{cases}
$$

But $(M(w \cdot \lambda): L(w \cdot \lambda))=1 \neq 0$, and the result follows.

COROLlaRY 5.5. Let $\lambda, \mu \in P^{+}, w_{1}, w_{2} \in W$. Then $\left(M\left(w_{1} \cdot \lambda\right): L\left(w_{2} \cdot \lambda\right)\right)=$ $\left(M\left(w_{1} \cdot \mu\right): L\left(w_{2} \cdot \mu\right)\right)$.

ProOF. By the theorem, both sides of the equation are equal to $\left(M\left(w_{1} \cdot 0\right)\right.$ : $\left.L\left(w_{2} \cdot 0\right)\right)$.

\section{REFERENCES}

1. V. V. Deodhar, O. Gabber, and V. G. Kac, Structure of some categories of representations of infinite-dimensional Lie algebras, Adv. in Math. 45 (1982), 92-116.

2. H. Garland and J. Lepowsky, Lie algebra homology and the Macdonald-Kac formulas, Invent. Math. 34 (1976), 37-76.

3. J. C. Jantzen, Möduln mit einem höchsten Gewicht, Lecture Notes in Math., vol. 750, SpringerVerlag, Berlin, 1979.

4. V. G. Kac, Simple irreducible graded Lie algebras of finite growth, Izv. Akad. Nauk SSSR 32 (1968), 1323-1367; English transl., Math. USSR-Izv. 2 (1968), 1271-1311.

5. __ Infinite-dimensional Lie algebras and Dedekind's $\eta$-function, Funktional. Anal. i Prilozhen. 8 (1974), 77-78; English transl., Functional Anal. Appl. 8 (1974), 68-70.

6. V. G. Kac and D. A. Kazhdan, The structure of representations with highest weight of infinitedimensional Lie algebras, Adv. in Math. 34 (1979), 97-108.

7. R. V. Moody, A new class of Lie algebras, J. Algebra 10 (1968), 211-230.

8. A. Rocha-Caridi and N. R. Wallach, Projective modules over graded Lie algebras. I, Math. Z. 180 (1982), 151-177.

Department of Mathematics, Clark University, WorCester, Massachusetts 01610 